\title{
Mobile adaptive crowd optimization scheme based on distance field
}

\author{
Ya-chun Tang ${ }^{1 *}$, Xiao-bo Guo ${ }^{2}$ and Xiang-dong Yin ${ }^{1}$
}

\begin{abstract}
For complexity and efficiency of the multi-objective optimization, we proposed the mobile distance field-driven adaptive crowd optimization algorithm. In space, we modify the surface parameters based on the corresponding changes of the distance field to obtain the moving target's moving track and moving surface. When the curve of the moving track is changed, the $x$ axis and the $y$ axis of the moving track are adjusted adaptively. In this paper, the moving process is divided into three processes: the target dynamic crowd control, the crowd model algorithm, and the predictive control of linear time domain based on the moving target prediction and crowd control algorithm. Then, the multi-objective optimization algorithm of moving objects is proposed by using the crowd model to predict the status and the position of the target. The experimental results show the high accuracy, low complexity, and high efficiency of the proposed optimization algorithm.
\end{abstract}

Keywords: Mobile crowd, Adaptive optimization, Distance field, Multiple objects

\section{Introduction}

Multi-objective optimization can be applied to a large number of practical applications [1]. The multi-objective optimization of the practical problems requires [2] simple architecture, easy implementation, low complexity, and low [3] and high optimization efficiency. However, the above features are often contradictory [4]. The search of a compromise between multiple characteristics [5] becomes the key to the development of multi-objective optimization applications.

The new subspace clustering algorithm was proposed by the authors of article [6], which segments the videos into consistent spatial-temporal regions with multiple classes. A novel algorithm was presented in article [7] that exploits joint optimization of representation and classification for robust tracking in which the goal is to minimize the least-squares reconstruction errors and discriminative penalties with regularized constraints. In article [8], a multi-objective particle crowd optimization technique is applied to a group of consecutive frames to reduce the number of branches in each tracking tree. The novel algorithm was presented in the article [9] for the removal of reflections generated by objects on reflecting floors. The block-coordinate Gauss-Newton/ regression method was proposed by Kim D S et.al [10], in order to conduct a correlation-based registration considering the intensity difference between images in the presence of outlier objects. The projection image generation algorithm [11] was proposed to design and fabricate a complex 3D scaffold, which automatically and robustly generates $2 \mathrm{D}$ projection image data. The author of article [12] proposed a repeated use of screened Poisson to compute a part coding and extracting distance field. A highly scalable method was proposed in article [13] for computing 3D distance fields on massively parallel distributed-memory machines.

In view of the above achievements and problems, based on the multi-objective optimization model, we proposed adaptive mobile crowd optimization based on distance field. The rest of the paper is organized as follows: Section 2 describes the moving target distance field analysis model. Section 3 proposes the adaptive mobile crowd optimization. The performance evaluation is shown in Section 4. Finally, the conclusions are given in Section 5.

\footnotetext{
* Correspondence: yachuntang@yeah.net

${ }^{1}$ School of Electronics and Information Engineering, Hunan University of

Science and Engineering, Yongzhou, Hunan 425199, China

Full list of author information is available at the end of the article
} 


\section{Moving target distance field analysis model}

In space, the moving track and the moving surface of the moving target have the following form:

$$
\left\{\begin{array}{c}
S_{C}=\left(\begin{array}{c}
x(m, n, t) \\
y(m, n, t) \\
z(m, n, t)
\end{array}\right) \\
T_{C}=\sum_{t=1}^{T} \frac{1}{2} a^{2} t+v_{0}
\end{array}\right.
$$

Here, $S_{C}$ represents the surface formed by the moving object. $T_{C}$ represents the moving trajectory of a moving target. The $t$ represents the moving time of the target. $T$ represents the total length of the moving target. Parameters $x, y$, and $z$ represent the three component of the three-dimensional space of the moving target. Parameter $V_{0}$ represents the initial movement speed. Parameter $a$ indicates the acceleration. Symbols $m$ and $n$ are the parameters of the moving surface. The moving surface is usually determined by the range of parameters. According to the formula (1), the parameters of the moving surface can be easily generated. In the form of moving surface parameters, the dot sequence of the moving surface is generated. We can get the moving surface by the linear processing of the store sequence and take the parameter value. Therefore, it is very convenient and efficient to form and update the moving surface. The time linearized by the moving surface has the characteristics of free rotation, fast state transition, and so on.

Moving surface has a good character of time and space, but it is a difficult problem to obtain the moving data parameters of a given object. Because the moving object in the three-dimensional space must be consistent with the curve of the moving surface. We consider the distance field to avoid the curved surface distortion of moving objects. The distance field is the moving surfaces between two adjacent samples of the moving target. When the shape of the curved surface changes, we modify the surface parameterization based on the corresponding changes in the distance field to keep the matching relationship. When the structure of the moving trajectory changes, we decompose the moving trajectories in the $x$ axis and the $y$ axis according to the formula (2).

$$
\begin{aligned}
& T_{C}=\left\{x\left(a, v_{0}\right), y\left(v_{0} t, a t+v_{0}\right)\right\} \mid \sum_{t=1}^{T} \frac{1}{2} a^{2} t+v \\
& \left\{\begin{array}{l}
T_{C}>\varepsilon: \text { Consistent }=1 \\
T_{C}=\varepsilon: \text { Consistent }=0 \\
T_{C}>\varepsilon: \text { Consistent }=2
\end{array}\right.
\end{aligned}
$$

Here, $\varepsilon$ is the threshold value of the curved surface. Parameter Consistent represents the degree of consistency between the moving objects in the three- dimensional space and the curvature of the moving surface. Number 1 indicates complete agreement. Number $O$ is not consistent, can be adjusted according to the distance field. Number 2 indicates that it is not consistent and cannot be adjusted.

The distance field function of the moving target is the key to the optimization of the moving target. For the moving surface, the distance field function defines the direction of the moving point to the moving target. The distance field function of the moving surface is given in Eq. (3), which is shown in Fig. 1.

$$
f(d)=\left\{\begin{array}{l}
d\left(p, S_{C}\right), \text { Consistent }=1 \\
\bar{d}\left(p, S_{C}\right), \text { Consistent }=0 \\
\bar{d}\left(p, S_{C}\right) \cdot F_{d}, \text { Consistent }=2
\end{array}\right.
$$

Here, $d\left(p, S_{C}\right)=\lim _{p \in S_{C}}\left(T_{C}(t) S_{C}(p)\right)$

Surface treatment would be finished based on the moving target trajectory data $P\left\{p_{i}\right\}_{t=1}^{T}$ with formula (1). The distance field function defined on the partition can make the matrix $S_{C}$ in the zero level. The state is able to approximate the distance field of the real-time data points of the moving target. This process can make the Consistent value of the data points of the moving target to be 1 . The Consistent value can be fitted by minimizing the following function as shown in Eq. (4).

$$
\min \sum_{i=1}^{n}\left\|\prod_{t=0}^{T} S_{C}(t) T_{C}(i)\right\|^{\varepsilon}
$$

In summary, Fig. 2 gives the moving target distance field analysis structure for moving target data surfaces.

\section{Adaptive mobile crowd optimization \\ 3.1 Crowd model of moving target}

Crowd model of the moving target is a linear fusion intelligent time domain mapping control model. The model

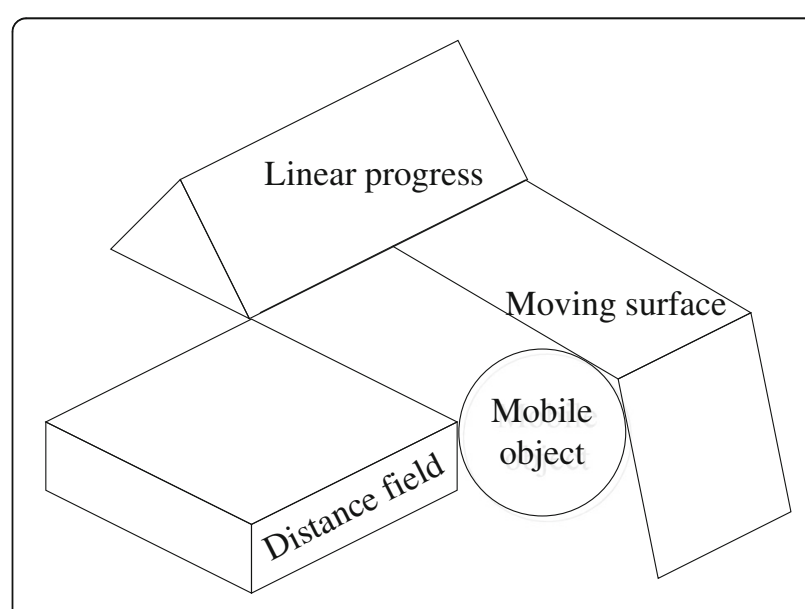

Fig. 1 Distance field function 

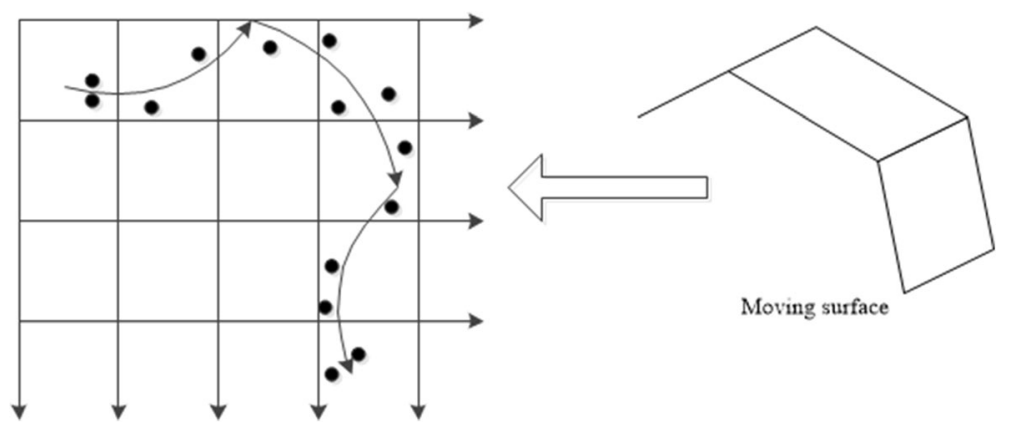

Fig. 2 Surface data point of moving target

is based on the cooperation of moving target prediction model and crowd control algorithm. The model includes the dynamic control, the crowd control model and the linear time domain predictive control. The crowd control can predict the status and position of moving objects by using crowd model. The crowd control model can design the mobile constraints and crowd optimization objectives based on the real-time mobile trajectory information. The model can generate the target state matrix and the target crowd information for the relationship mapping. The realtime continuous optimization is realized by crowd control with output information. The crowd algorithm can transform the tracking problem of moving objects to the target of the crowd optimization problem. This conversion is realized by using the crowd information of each moving target in the time domain to reset the optimization objective and the real-time optimal control conditions.

As shown in Fig. 3, the moving target prediction, optimization, and crowd optimization model can be realized by the mobile location and crowd control scheme. The moving target crowd models are extended to the control problem of the moving optimization of the mapping object according to the prediction, optimization, and correction. The model can be used to the trajectory control of moving objects and be suitable for the real-time decision of the upper control center.

The controller of moving target crowd model is composed of crowd model and mapping model. The two submodels can be used to predict the status and position of moving targets in real time according to the moving trajectory and the moving surface. The model can provide the conditions for crowd optimization of moving objects by the objective state of different time domain.

The moving state of the moving object in the $t$ time and the control state of the crowd control are shown in formula (5). Here, parameter $L$ represents the crowd state transition matrix of the moving object. According to the linear feature in time domain, the output of the moving target module is $O((s, l) \mid t)$. The crowd optimization control weight is $\varphi$.

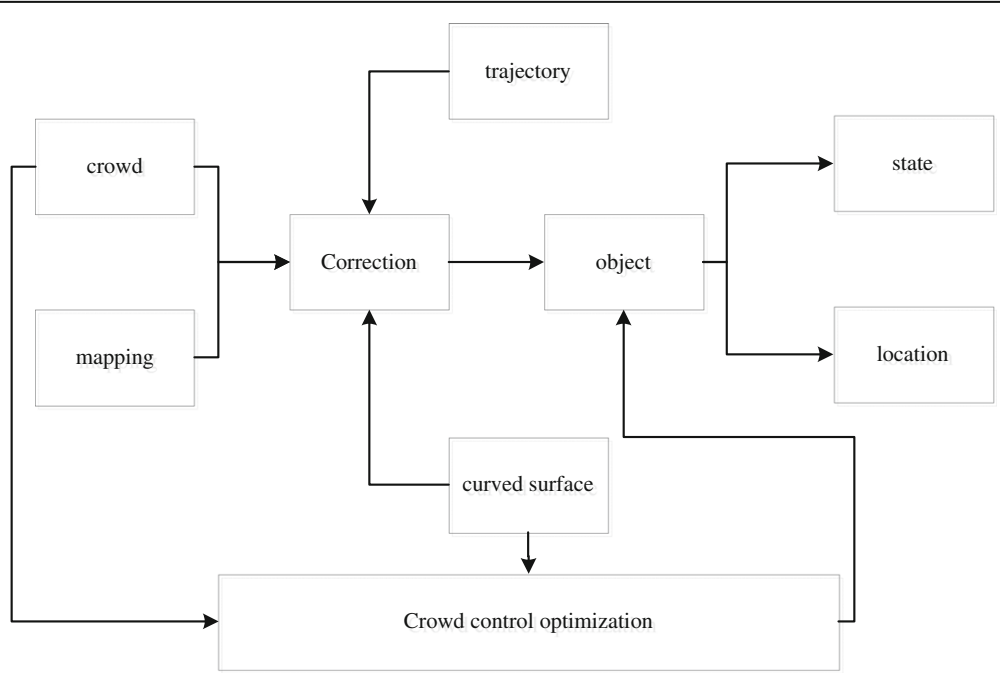

Fig. 3 Architecture of moving target crowd model 


$$
\left\{\begin{array}{l}
O((s, 1) \mid t)=\frac{L \cdot \sum_{t \rightarrow T} \varphi f(t)}{\int S_{C}(t)} \\
O((1, l) \mid t)=\frac{L \cdot \sqrt{\sum_{t \rightarrow T} \varphi f(t)}}{\int T_{C}(t)}
\end{array}\right.
$$

According to the position of the moving target, the crowd distribution of time domain objects is predicted. The output state vector of the model is used to monitor the trajectory of moving objects and the moving surfaces. The objective optimization condition is provided according to the adjustment of crowd weight and change of linear time domain.

\subsection{Adaptive multi-objective optimization}

The multi-objective optimization problem of moving objects can be described as:

$$
\left\{\begin{array}{l}
\min _{t \in T} f(O)=[f(O((0,0) \mid t)) \quad \cdots \quad f(O((s, l) \mid t))] \\
\text { s.t.s. } s_{t}(O)_{s \rightarrow S, l \rightarrow L} \\
L_{t}(O)_{s \rightarrow S, l \rightarrow L}
\end{array}\right.
$$

When $S_{C}(t)=0$ and $T_{C}(i)=0$, by crowd state transfer and linear mapping, the optimization objective is mapped to the moving trajectory and the moving surface, such as formula (7).

$$
\left\{\begin{array}{l}
s_{t}(O)_{s \rightarrow S, l \rightarrow L} \leq \varphi S_{C}(t)_{t \rightarrow T} \\
L_{t}(O)_{s \rightarrow S, l \rightarrow L} \leq \varepsilon T_{C}(i)_{i \rightarrow n}
\end{array}\right.
$$

Thus, the multi-objective optimization formula is equivalent to

$$
\left\{\begin{array}{l}
\min _{t \in T} f(O)=[f(O((0,0) \mid t)) \quad \cdots \quad f(O((s, l) \mid t))] \\
\text { s.t. }\left\llcorner\varphi S_{C}(t)_{t \rightarrow T}\right\rfloor \\
\left\lceil\varepsilon T_{C}(i)_{i \rightarrow n}\right\rceil
\end{array}\right.
$$

Adaptive multi-objective optimization algorithm of crowd process is shown in Fig. 4. Algorithm procedure is described as follows:

Step1. Select the appropriate sampling time. Determine the moving target. Speed and location of sampling are initialized randomly in an optimized range.

Step2. Get the surface by the moving objects in the moving process. Track of moving target is obtained. Tracing the time of moving targets. Get the total length of the moving time. Construction of threedimensional spatial component of moving objects. Get the initial movement speed. Obtain the acceleration of

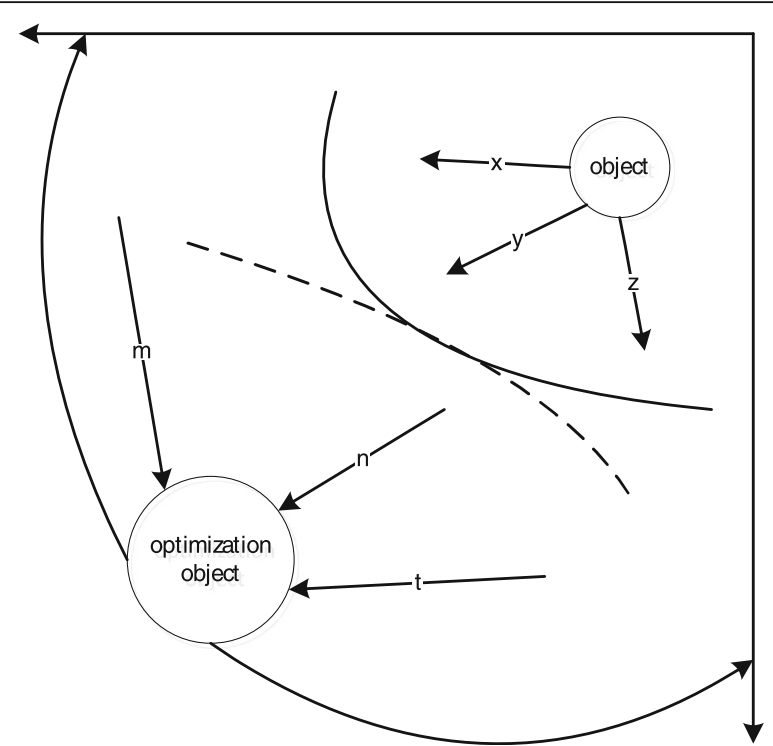

Fig. 4 Process of adaptive multi-objective crowd optimization algorithm

moving target according to the track and speed. Initialize of the parameters of the moving surface. Step3. Select the state and position of the moving surface.

Step4. For each moment in the moving trajectory, the distance field function is obtained according to the formulas (2) and (3). According to this function, the velocity and position of the moving target are updated, and the new moving trajectory is obtained.

Step5. For each data point of the moving surface, the optimization goal would be set according to the method of distance field function shown in Fig. 1. Step6. According to the method of Fig. 3, based on mobile positioning and crowd control, crowd optimization model is obtained for predicting and optimizing the moving goal, as well as the crowd acquisition.

Step7. The real-time decision of the upper control center is supported by mobile target trajectory control from mobile crowd multiple target optimization. Step8. According to formula (6), the multi-objective optimization model of moving objects is obtained.

According to Fig. 4 and formula (5), step 3 realizes the adaptive multi-objective optimization.

\section{Performance evaluation}

The five optimization functions are selected to describe the moving objectives. The functions F1, F2, and F3 are as shown the formula (9). The test functions F4 and F5 are shown in the formula (10). Here, the functions F1, F2, and F3 are the main points of the optimal control of the moving crowd target. The functions F4 and F5 have 
the characteristics of movement shown in Fig. 1. Different parameters are selected for different functions. The test parameters are shown in Table 1.

$$
\left\{\begin{array}{r}
\min f(O)=O(s, l)_{t} \\
\text { s.t. } S_{C}^{\varepsilon}=\varphi S_{C}(t)_{t \rightarrow T} \\
T_{C}^{\varepsilon}=\varphi T_{C}(i)_{i \rightarrow n}
\end{array}\right.
$$

$$
\left\{\begin{array}{l}
\text { minf }(O)=O(s, l)_{t} \\
\min f(x, y, z)=\prod_{t}^{T} O_{x, y, z} \\
\text { s.t. } O((1,0) \mid t)=L_{1} \cdot \sum_{t \rightarrow T} \varphi f(t) \\
O((0,1) \mid t)=L_{0} \cdot \sum_{t \rightarrow T} \frac{\varepsilon f(t)}{\int S_{C}(t)}
\end{array}\right.
$$

Figure 5 shows the ACO-DF (mobile adaptive crowd optimization scheme based on distance field) algorithm and the positioning error of the target optimization algorithm based on mobile search denoted as OO-MS. ACO-DF algorithm tracks the moving surface in the time domain and spatial domain. ACO-DF algorithm considers the distance field to avoid the distortion of the curved surface of the moving target. The moving object of the three-dimensional space is consistent with the arc structure of the moving surface. Positioning error is obviously less than OO-MS algorithm. In particular, the ACO-DF algorithm can effectively reduce the influence of the moving speed on the localization by modifying the surface parameters based on the corresponding change of the distance field.

Figure 6 shows the ACO-DF algorithm and OO-MS algorithm to optimize the number of iterations. Based on the cooperative control of the moving target prediction and crowd control algorithm, the ACO-DF algorithm can realize the intelligent time domain mapping control based on linear fusion. In addition, the ACO-DF algorithm obtains the state and the position of the moving target by using the crowd to control the dynamic and predictive control of the moving object. So, the number of iterations of the ACO-DF algorithm is

Table 1 Testing parameters

\begin{tabular}{llll}
\hline Parameter & Value & Parameter & Value \\
\hline$T$ & $10 \mathrm{~m}$ & $x$ & $200 \mathrm{~m}$ \\
$y$ & $200 \mathrm{~m}$ & $z$ & $100 \mathrm{~m}$ \\
$a$ & $5 \mathrm{~m} / \mathrm{s}$ & $V_{0}$ & $2 \mathrm{~m} / \mathrm{s}$ \\
$d$ & $10 \mathrm{~m}$ & $d_{T H}$ & $20 \mathrm{~m}$ \\
$\varphi$ & 0.3 & $\varepsilon$ & 0.01 \\
\hline
\end{tabular}

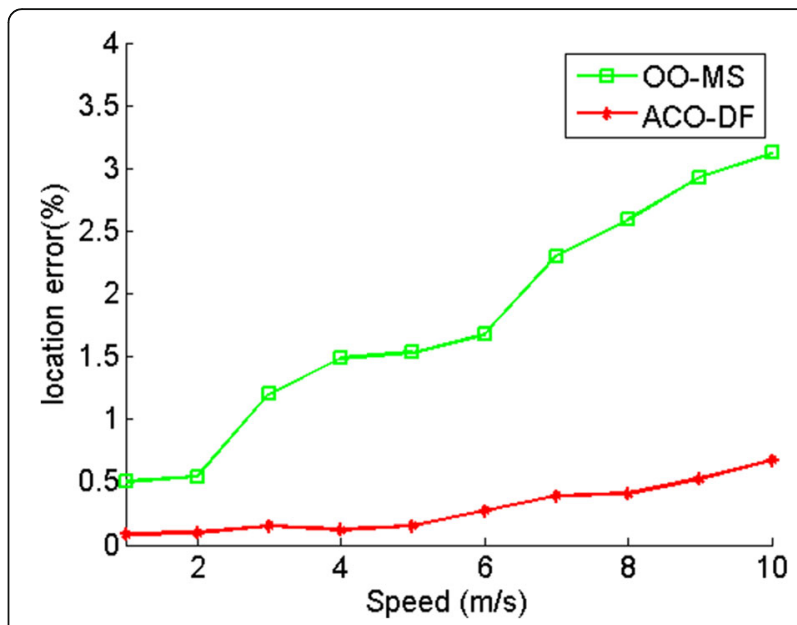

Fig. 5 Location error

significantly less than that of the OO-MS algorithm. ACO-DF algorithm based on trajectory information to design a real-time movement restriction and crowd optimization goal is generated for mapping relationship between target state matrix and the target group of metal information. In this way, the area of the moving surface is small, and it is helpful to improve the efficiency and accuracy of multi-objective optimization, which are shown as Fig. 7.

\section{Conclusions}

We present the distance field-driven adaptive mobile goals in intelligent optimization algorithms to solve the multi-objective optimization complexity and efficiency issues. First, based on the corresponding changes in the distance field, we modify the surface parameters in the airspace. In this way, the moving track and surface of the object can be obtained. Secondly, based on the curve

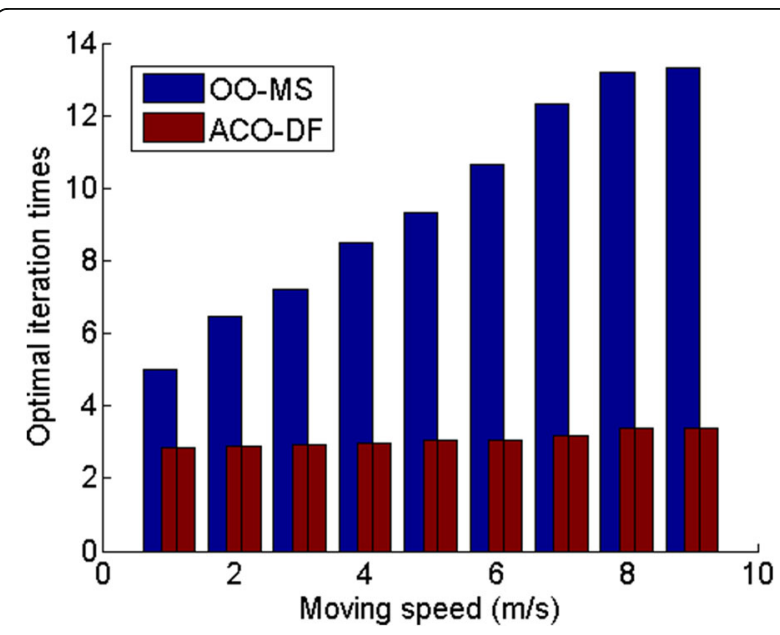

Fig. 6 Optimal iteration times 


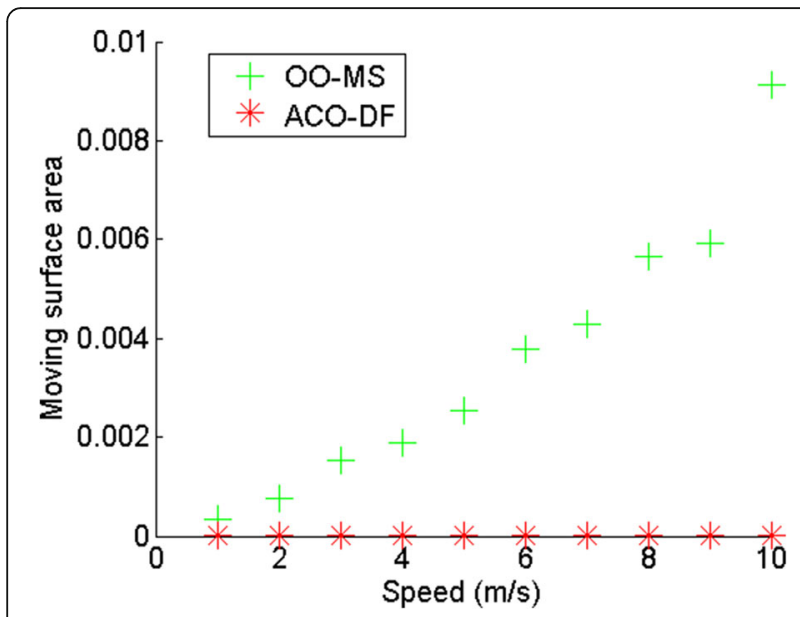

Fig. 7 Moving surface area

structure of the moving track, we adaptively adjust the $x$ axis and the $y$ axis structure of the moving trajectory. Then, we decompose the process into three processes, which are target dynamic crowd control, crowd model algorithm control and linear time domain predictive control. The three processes are based on the cooperative control of the moving target prediction and crowd control algorithm to form a multi-objective optimization framework. Finally, the multi-objective optimization algorithm of moving objects is proposed by using the crowd model to predict the status and the position of the target. The results of the five test functions show that the proposed algorithm has higher localization accuracy, lesser iteration times, and smaller surface area than the target optimization algorithm based on mobile search.

\section{Acknowledgements}

This work is supported in part by the Hunan Provincial Department of Education Scientific Research Outstanding Youth Project (14B070) and Science and Technology project of Hunan Province (2014FJ6095).

\section{Competing interests}

The authors declare that they have no competing interests.

\section{Author details}

${ }^{1}$ School of Electronics and Information Engineering, Hunan University of Science and Engineering, Yongzhou, Hunan 425199, China. ${ }^{2}$ College of Computers, Henan University of Engineering, Zhengzhou 451191, China.

Received: 4 August 2016 Accepted: 2 November 2016

Published online: 15 November 2016

\section{References}

1. G Zhu, Z Wang, G Zhang, Research on a combined device SMES-SFCL based on multi-object optimization[J]. IEEE Trans. Appl. Supercond. 15(2), 2019-2022 (2005)

2. J Liu, L Cao, Z Li et al., Plane-based optimization for 3D object reconstruction from single line drawings.[J]. IEEE Trans. Pattern Anal. Mach. Intell. 30(2), 315-327 (2008)

3. APD Poz, RAB Gallis, JFCD Silva, Three-dimensional semiautomatic road extraction from a high-resolution aerial image by dynamic-programming optimization in the object space[J]. IEEE Geosci. Remote Sens. Lett. 7(4), 796-800 (2010)
4. P Fungtammasan, T Watanabe, Grasp input optimization taking contact position and object information uncertainties into consideration[J]. IEEE Trans. Robot. 28(5), 1170-1177 (2012)

5. J Stefanski, B Mack, B Waske, Optimization of object-based image analysis with random forests for land cover mapping[j]. Sel. Top. Appl. Earth Obs. Remote Sens. IEEE J. 6(6), 2492-2504 (2013)

6. C Wang, Y Guo, J Zhu et al., Video object co-segmentation via subspace, clustering and quadratic pseudo-Boolean optimization in an MRF framework[J]. IEEE Trans. Multimedia 16(4), 903-916 (2014)

7. Q Wang, F Chen, W Xu et al., Object tracking with joint optimization of representation and classification[J]. IEEE Trans. Circuits Syst. Video Technol. 25(4), 638-650 (2015)

8. K Ahmadi, E Salari, Small dim object tracking using a multi objective particle swarm optimisation technique[J]. IET Image Process. 9(9), 820-826 (2015)

9. D Conte, P Foggia, G Percannella et al., Removing object reflections in videos by global optimization[J]. IEEE Trans. Circuits Syst. Video Technol. 22(11), 1623-1633 (2012)

10. DS Kim, K Lee, Block-coordinate Gauss-Newton optimization and constrained monotone regression for image registration in the presence of outlier objects[]]. IEEE Trans. Image Process. 17(5), 798-810 (2008)

11. DJ Yoo, Advanced projection image generation algorithm for fabrication of a tissue scaffold using volumetric distance field[J]. Int. J. Precis. Eng. Manuf. 15(10), 2117-2126 (2014)

12. M Genctav, A Genctav, S Tari, Nonlocal via local-nonlinear via linear: a new part-coding distance field via screened Poisson equation[J]. J. Math. Imaging Vision 55(2), 242-252 (2015)

13. H Yu, J Xie, KL Ma et al., Scalable parallel distance field construction for large-scale applications[]]. IEEE Trans. Vis. Comput. Graph. 21(10), 1187-200.s (2015)

\section{Submit your manuscript to a SpringerOpen ${ }^{\circ}$ journal and benefit from:}

- Convenient online submission

- Rigorous peer review

- Immediate publication on acceptance

- Open access: articles freely available online

- High visibility within the field

- Retaining the copyright to your article

Submit your next manuscript at $>$ springeropen.com 